\title{
DYNAMIC VISUAL SERVO CONTROL OF ROBOTS: AN ADAPTIVE IMAGE-BASED APPROACH'
}

\author{
L. E. Weiss - A. C. Sanderson - C. P. Neuman
}

\author{
The Robotics Institute \\ Carnegie-Mellon University \\ Pittsburgh, PA. 15213
}

\begin{abstract}
Sensory systems, such as computer vision, can be used to measure relative robot end -effector positions to derive feedback signals for control of end-effector positioning. The role of vision as the feedback transducer affects closed-loop dynamics, and a visual feedback control strategy is required. Vision-based robot control research has focused on vision processing issues, while control system design has been limited to ad-hoc strategies. We formalize an analytical approach to dynamic robot visual servo control systems by first casting position-based and image-based strategies into classical feedback control structures. The image-based structure represents a new approach to visual servo control, which uses image features (e.g., image areas, and centroids) as feedback control signals, thus eliminating a complex interpretation step (i.e., interpretation of image features to derive world-space coordinates). Image-based control presents formidable engineering problems for controller design, including coupled and nonlinear dynamics, kinematics, and feedback gains, unknown parameters, and measurement noise and delays. A model reference adaptive controller (MRAC) is designed to satisfy these requirements.
\end{abstract}

\section{Int roduction}

Flexible robotic systems should provide a capability to automatically modify positions and trajectories to accommodate changes in the task or task environment. Such flexibility is achievable only through the integration of sensors into robotic systems, and visual sensing, in particular, provides fundamental capabilities in the adaptation of robots to both unstructured and precision tasks. The promise of fast vision systems and special purpose high-speed optical sensors would permit the synthesis of dynamic "visual servo control systems." In this paper, we describe a strategy which integrates visual sensing with robot movements, and will emphasize the design of such a "visual servo control system" in order to achieve stable and predictable system dynamics.

Vision systems can be used as transducers to measure the relative positions between robot end-effectors and objects in the robot's work space. The interpretation of two dimensional image information to estimate positions normally requires the

\footnotetext{
"This research has been supported in part by the Westinghouse Electric Corporation and in part by the Robotics Institute of Carnegie-Mefion University.
}

extraction of quantitative image features which are related to stored object models. The positional error signal, between reference and measured positions, may be used to design a "position-based" visual servo control system ${ }^{1}$. In our research we have explored an alternative strategy ${ }^{2}$. Image features which are monotonically related to spatial position may be used as a basis for control in place of position estimates. These features typically include structural components of the image such as points, lines, and areas, as well as quantitative parameters attached to them. Such an "image-based" visual servo control strategy may offer advantages in terms of reduced delay and estimation noise, and provide a convenient means for robot task training. However, image-based control requires special attention to control strategies which accommodate position-to-feature relationships.

In dynamic visual servo control systems the role of computer vision as the measurement process affects the overall system dynamics, and a visual feedback controller is required for stability and to achieve a suitable transiont response. The linearity, noise properties, coupling, and computational delays of this measurement process become essential considerations for controller design. Formal analysis and design of feedback controllers for visual servoing, in terms of well-defined control theory, has not appeared in literature except for a simple case ${ }^{3}$. Visual servo controllers have been designed using ad-hoc strategies ${ }^{4}$. In our research, we have organized previously implemented and proposed visual servo control strategies into clearly defined feedback control structures, and clarify the requirements placed on the visual servo controller ${ }^{5}$.

In position based approaches to visual servo control, the image interpretation step can be complex and affect control system design and performance by adding additional time delays, and introducing measurement noise resulting from inaccuracies of the object and transduction modeling. Image features are often coritinuous functions of object position, and for fixed ranges of control, provide sufficient information for robot control without carrying out the interpretation process. An image-based system is described in more detail below.

The measurement process in a position-based visual servo structure can be decomposed into two nonlinear transformations. First, the transduction and feature extraction functions, or world-to-feature space transformation, can be viewed as the inverse of an ideal interpretation, in the absence of noise, according to: 


$$
f=r^{-1}\left[\underline{x}_{\text {rel }}\right]
$$

where 1 is "ideal" in the sense of being based on exact models, and $\underline{\underline{X}}_{r e t}$ is a relative end-effector position. Second, the features are mapped to world space by the interpretation transformation:

$$
\hat{\mathrm{x}}_{\mathrm{Tel}}=\hat{\imath}[\mathrm{g}]
$$

If the ideal interpretation has a unique inverse mapping, over the control region of interest, such that $\underline{\underline{X}}_{r e l}$ are single-valued functions of $\mathbf{f}$, then this suggests that the system can be controlled, to unique end-points, using features directly as the feedback and reference signals, thus eliminating the interpretation step. The uniqueness condition is satisfied, for the control region of interest, when ${ }^{6}$ :

\section{The first partial derivatives of $\mathfrak{f}$ are continuous, and}

2. If the Jacobian of the ideal inverse interpretation is nonsingular; i.e.,

$$
\operatorname{det} \frac{\partial r^{-1}\left[\underline{x}_{\text {rel }}\right]}{\partial \underline{\underline{x}}_{\text {rel }}} \doteq \operatorname{det}\left[J_{\text {feat }}\right] \neq 0
$$

where $\mathrm{J}_{\text {feat }}$ is defined as the feature sensitivity matrix.

In practice, $J_{\text {feat }}$ could be estimated on-line to test the condition in eq.(3). This condition must be true for both position and image-based approaches. Further, since the determinant is only defined for square matrices, then the permitted number of degrees-of-freedom must equal the number of measured features.

A digitally controlled dynamic image-based visual servo (IBVS) control structure, which uses feature feedback, is represented in Figure 1.1. This system was first first proposed by Sanderson and Weiss ${ }^{5}$. In Figure 1-1, $\underline{u}$ are the control signals, $\underline{q}$ are the robot joint coordinates, and $n_{d}$ is the number of feedback delays introduced by the vision processing. The characteristics of each block are described in the next section. The reference signal $f_{\text {ref }}(k)$ can be defined directly in image feature space using a "teach-by showing" strategy whereby an image is transduced in the desired reference position and the corresponding extracted features represent the reference feature values. In the "teachby-showing" approach it is assumed that the inverse transformation $\mathrm{l}^{-1}$ is unknown. Therefore, the controller $\mathrm{C}$ must be based on a design approach which not only compensates for the nonlinear and coupled properties of $\mathrm{r}^{-1}$, but also for unknown values.
The image-based structures are representative of sensor based control structures which include:

1. A measurement system which generates sensory information that is difficult to interpret;

2. A sensory feedback path which can be nonlinear, coupled, noisy, contain unknown parameters, and include delays; and

3. A robot under control which can be dynamically and kinematically nonlinear and coupled.

A control approach for image-based systems may then have potential for application to other sensory modalities (e.g., force or acoustic). The potential and the novelty of this approach, lead us to perform extensive analysis, design and evaluation of "teach-by-showing" image-based systems ${ }^{2}$.

\section{Control of Image-Based Systems}

Several characteristics of image-based structures present particular challenges for design of a suitable controller. In addition to compensating for robot dynamics, measurement noise, and $n_{d}$ feedback delays, the feedback controller, $C$, must also compensate for the nonlinear and coupled feature transformations. The relationships between Cartesian paths and feature trajectories must also be considered in the selection of feature reference signals.

To design a controller, it is useful to first consider the smallsignal model (about a nominal operating point or trajectory) of an the IBVS structure in Figure 1-1. The control signals are applied through digital-to-analog converters (DAC) which can be modeled by the cascade of an ideal impulse sampler and a zero-order hold with a saturation nonlinearity ${ }^{7}$. The system output is the undelayed feature, while the feedback path is modeled by discrete unit delays. Linearized open-loop robot dynamics $^{8}$, or equivalent linear $1 / 0$ models ${ }^{9}$, are represented by the discrete-time $Z$-transformation $\mathrm{W}_{\mathrm{p}}\left(\mathrm{z}^{-1}\right)$. The feedback path is characterized by an overall small-signal sensitivity matrix $\mathbf{J}$ given by

$$
\mathbf{J} \doteq \mathbf{J}_{\text {feat }} \mathbf{J}_{\text {arm }}
$$

where $\mathbf{J}_{\text {arm }}$ is the arm Jacobian. In addition to the control requirements of the robot dynamics the design of the controller $C$ also depends on the " $J$ " sensitivity matrices, feedback

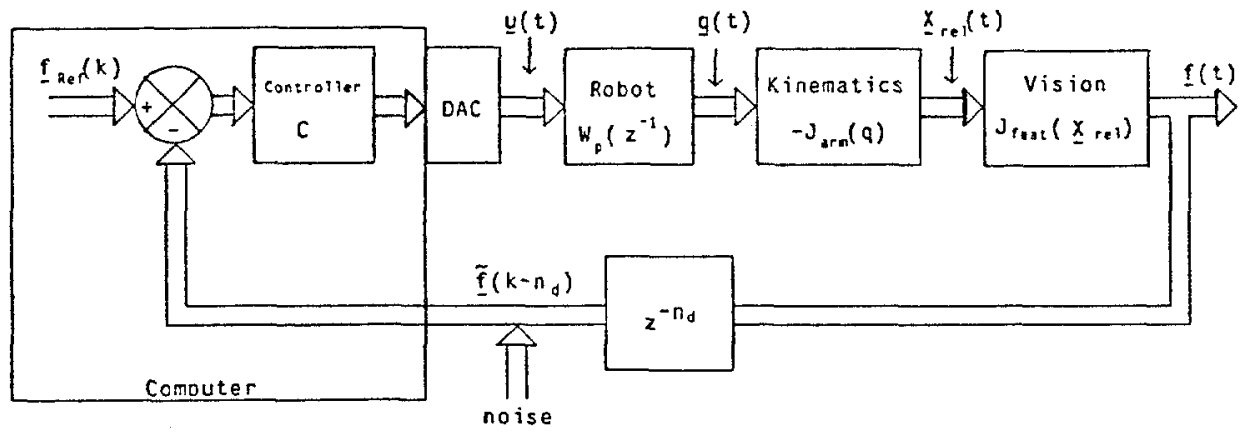

Figure 1-1: Image-Based Visual Servoing 
delays, and measurement noise. The sensitivity matrices are nonlinear and coupled functions of $\underline{g}$ and $\underline{X}_{\text {rel }}$ i thus, $\mathrm{J}$ varies as q varies, and feature-space transformations are manifested by time-varying open-loop gains. Predicted values of $\mathrm{J}$ can deviate from actual values due to inaccuracies in the modeling of the three dimensional object and traisduction process, and from drift and variation in the transducer parameters. At the extreme, the values may be completely unknown apriori when minimal knowledge of the inverse interpretation transformations $\mathrm{l}^{-1}$ are available, such as arises when task programing is limited to the "teach-by-showing" strategy. Fixed feedback controller designs have limitations in the control of such nonlinear and unknown systems. Even if the nonlinearities are known, a fixed controller design for these systems is a formidable engineering problem. In contrast, an adaptive approach to controller design appears to be applicable for these requirements. The IBVS controller design used in our research therefore emphasizes the adaptive approach.

\subsection{Adaptive IBVS Control}

In the context of joint-level control, researchers have recently begun to evaluate the applications of adaptive control to robotic manipulators $8,10,11,12,13,14$. Adaptive control has the potential to compensate for parameter uncertainty and variation over a wide range, while operating at high joint speeds. In these adaptive control schemes, an adjustable controller uses on-line identification to identify parameters of an equivalent input/output $(1 / 0)$ linear model of the robot based on the 1/O information vectors $\underline{u}(k)$ and $g(k)$ (actuator control signals and joint positions respectively), under the assumption that the robot is linear and constant, but has unknown parameters. An equivalent $1 / 0$ model is one that predicts the output $g(k)$ from past and present $1 / O$ information independent of the physical model of the robot. The estimated parameter values are then used in a linear feedback controller as though they where the actual parameters.

The mathematical basis for our adaptive controller follows the enhanced identification error model reference adaptive control (MRAC) developed by Morris and Neuman ${ }^{15}$. While similar approaches have appeared in literature, their research focused on details of physical implementation including control signal saturation and controller stability, measurement noise, and computational complexity for microprocessor implementation. Since the algorithm did not include control of systems with discrete measurement delays, we have extended it to include control of systems with delay ${ }^{2}$. Additional modifications for applying uncoupled MRAC to the control of coupled nonlinear systems were also developed.

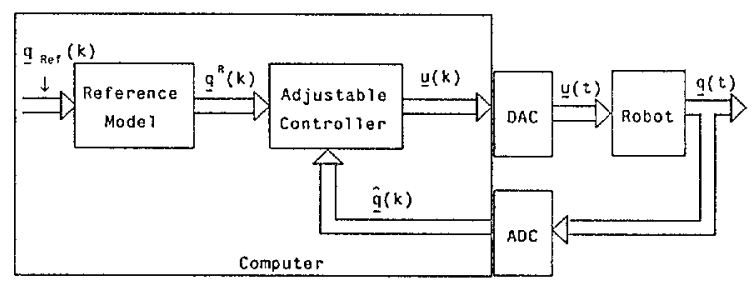

Figure 2-1: Model Reference Adaptive Control
In joint-level MRAC control (Figure 2-1), the reference modei output $\mathrm{q}^{R}(\mathrm{k})$ specifies a stable and realizable closed-loop dynamic response of the the output $g(k)$ to the reference signal $\mathrm{g}_{\mathrm{Ref}}(\mathrm{k})$. The difference between the reference model output and the process is called the full-parallel (FP) output error:

$$
\underline{\mathrm{e}}_{\mathrm{f}}^{\mathrm{F}}(\mathrm{k})=\underline{q}^{\mathrm{R}}(\mathrm{k}) \cdot \mathrm{g}(\mathrm{k}) \text {. }
$$

The adjustable controller utilizes the identified parameters information to adjust the gains on-line to drive the FP error to zero, thus forcing the robot output to track the reference signal in accordance with the performance specified by the model. In the identification-error method of MRAC control ${ }^{14}$ an identifier predicts the robot joint outputs, $t(k)$, based upon parameter estimates of an equivalent linear $1 / O$ model. The identification error

$$
\underline{e}_{1 D}(k)=g(k)-t(k)
$$

drives the adjustment mechanism which updates the estimates of the equivalent parameters. These estimates are then used to adjust the gains of a linear controller which is driven by the model output. The adjustment mechanisms can be designed from either parametric optimization or stability viewpoints.

Both single-input single-output (SISO) and multiple-input multiple-output (MIMO) equivalent model formulations can be used to derive the adaptive controller. In the context of jointlevel control, Neuman and Stone ${ }^{15}$ have justified the latter modeling approach by demonstrating that individual joints of a coupled and nonlinear robot can be modeled by linear time. varying second-order SISO transfer functions. They show that the transfer function parameters vary smoothly in the work space as a function of the joint positions, velocities, and accelerations. While coupled, or MIMO, controllers have an inherently greater potential for being able to uncouple a coupled system they have several potential disadvantages, including computational complexity and they do not lend themselves to modularity. A modular system can easily be extended to increasing degrees-of-freedom, and distributed processing. Uncoupled adaptive controllers have already demonstrated the potential to control dynamically coupled robots $^{13,14}$, and would be easier to implement in current laboratory and factory computing environments. For these reasons, the approach which we developed emphasized uncoupled control of coupled systems, using the concept of equivalent SISO plants. For example, a two degree-of-freedom (DOF) IBVS system is controlled by independent MRAC controllers in Figure 2-2.

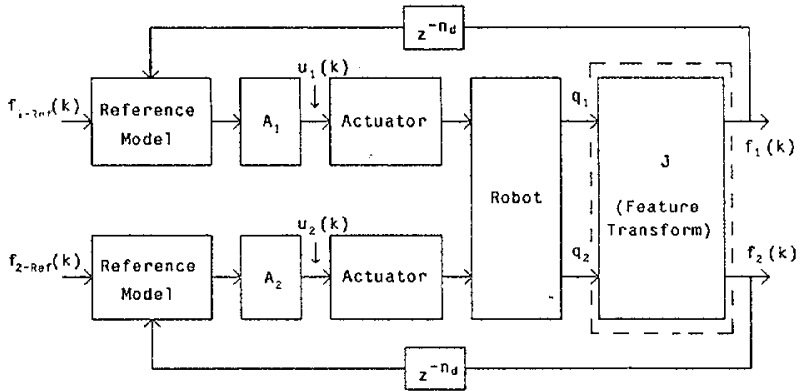

Figu re 2-2: MRAC Control of an IBVS System 


\subsection{Feature Selection and Assignment}

Feature transformation coupling (i.e., represented by the small-signal feature sensitivity matrix J) leads to related problems of feature selection and assignment. The feature selection process asks the question: How should a subset of $n$ features be selected from a set of $m$ possible control features $f$ $(i=1, \ldots, m)$, where $m>n$ ? And, the feature assignment process asks: Since uncoupled controllers are used to control coupled plants, which feature should be used to control each actuator? Both issues are related to the degree-of-coupling of the feature transformation. We have developed a measure of coupling to help answer these questions.

\subsubsection{Feature Assignment Using Diagonal Dominance}

In applications where uncoupled controllers are used to control coupled plants, there is always the problem of choosing which servo error will control which actuator; that is, for a set of $n$ outputs $y_{i}(i=1, \ldots, n)$, which servo error, $\Delta y_{i}$, should be filtered and coupled to the $j^{\text {th }}$ actuator as $u_{i}$ ? The control engineer tackles this problem with insight into the physical nature of the system and formulates qualitative notions of the degree-ofcoupling between the independent actuators and the system output. For joint-level robot control, $\Delta \mathrm{q}_{\mathrm{i}}$ should be used to derive $u_{i}$. For other systems, such as IBVS control, the assignment may not be straightforward. To formalize this procedure let the open-loop linear system be defined by

$$
\underline{Y}(s)=H(s) \underline{u}(s)
$$

where $H(s)$ is an $(n \times n)$ transfer function matrix. When the system is uncoupled, $\mathrm{H}(\mathrm{s})$ can be transformed into a diagonal matrix by switching the $j^{\text {th }}$ and $k^{\text {th }}$ columns of $H(s)$, and therefore the $j^{\text {th }}$ and $k^{\text {th }}$ rows of $\underline{u}(s)$, until all off diagonal elements of $\mathbf{H}(s)$ are zero. When $H(s)$ is diagonal, the only choice for servo error/actuator assignment is $u_{i} \leftarrow \Delta y_{i}$. When the system is coupled, then $H(s)$ cannot be transformed into a diagonal matrix. Servo error/actuator assignment selection can be accomplished by organizing $H(s)$ in a "diagonally dominant" fashion ${ }^{17}$, such that the diagonal elements dominate the offdiagonal elements. Diagonal dominance is defined as

$$
\left|H_{i j}(s)\right|>\sum_{\substack{j=1 \\ j \neq i}}^{n}\left|H_{i j}(s)\right| \quad \text { for } i=1, \ldots n
$$

When $\mathrm{H}(\mathrm{s})$ can be organized according to this definition of dominance, then limited stability properties of both coupled and uncoupled fixed control of the system can be formulated ${ }^{17}$. When applied to image based systems, with IJW $\mid \leftarrow H$ (s), we have shown that the sensitivity matrices cannof in general satisfy this definition of dominance.

An alternative approach is to organize $\mathrm{JW}_{\mathrm{p}}$ to maximize the inequality (8) over all possible column arrangements. This criterion reduces to defining the dimensionless measure of diagonal dominance as

$$
D(k)=\log \sum_{i=1}^{n} \sum_{j=1}^{n} \frac{\left|J W_{p-i j}(k)\right|}{\left|J W_{p-i j}(k)\right|}
$$

and then minimizing $D\left(J W_{p}\right)$ over all $n$ ! possible column arrangements. The logarithm of the dominance is used since the ratios change by orders of magnitude.

\subsubsection{Feature Selection}

The image of a typical scene generally contains more features than there are degrees-of-freedom to control. The number of features must equal the number of degrees-of-freedom in an image based system since the feature sensitivity matrix is constrained to be square. The possible number of ordered candidate feature subsets is

$$
p(m, n)=\frac{m !}{(m \cdot n) !}
$$

where ordering is required to consider the feature/joint assignment.

To arrive at a criterion for feature selection, two aspects of feature-based control are analyzed:

1. Ability to specify world space path using feature based trajectories (assuming that the control system can achieve a specified feature space performance), and

2. The control effort required to achieve the specified feature space dynamic performance.

It is shown below that the attributes of the feature sensitivity matrix, $J_{\text {feat }}$, relate to path performance, while the attributes of $J W_{p}$ relate to the control effort aspects.

With respect to world space path, it is desirable to be able to control independently each world level DOF. To achieve this goal, an ideal subset of features should yield a feature sensitivity $\mathrm{J}_{\text {feat }}$ which is diagonal and constant. Then,

$$
\Delta X_{i}=\frac{\Delta f_{i}}{J_{\text {feat }-i, i}}
$$

where, $\Delta \mathrm{X}_{i}$ is the path error for the $\mathrm{i}^{\text {th }}$ DOF, $\Delta \mathrm{f}_{i}$ is the $\mathrm{i}^{\text {th }}$ feature error, and $J_{\text {feat }-i, i}$ is the $(i, i)^{\text {th }}$ element of $J_{\text {feat }}$ If straight-line motion is desirable, and all of the features exhibit the same dynamic response, then straight-line motion would be achieved. For example, assume that the $i^{\text {th }}$ feature response is specified by the critically damped response

$$
f_{i}^{\circ}(t) \doteq f_{i}(t)-f_{i}(0)=\Delta f_{i}\left(1-e^{-t / \tau}\right)
$$

and all feature responses have the same time constant $\tau$. The response of the $i^{\text {th }}$ DOF is

$$
X_{i}^{0}(t) \doteq X_{i}(t)-x(0)=J_{\text {feat }-i, i}^{-1} f_{i}^{0}(t)
$$

The relationship between any two Cartesian degrees-offreedom becomes

$$
-\frac{X_{i}^{0}}{X_{j}^{0}}=\frac{J_{\text {feat }-\mathrm{i}}^{-1} \Delta f_{i}}{J_{\text {feat }-j}^{-1} \Delta f_{j}} \frac{1-e^{-t / \tau}}{1-e^{-t / r}}=\text { Constant }
$$

which is constant and specifies the equation of a straight-line in Cartesian coordinates. It thus becomes straightforward to specify straight-line motion.

If an ideal feature sensitivity matrix could be synthesized, then it still remains to control dynamically the system to achieve the 
desired feature response. Attributes of the overall sensitivity, $\mathrm{JW}_{\mathrm{p}}$, can be used to describe the control effort required to achieve the desired response. Similar to the feature sensitivity attributes, the idealized overall sensitivity matrix should be diagonal and constant. Diagonalization permits the unqualified use of independent SISO controllers. In our experience, these idealized sensitivity attributes cannot be expected in practice. The degrees-of-freedom are coupled and the sensitivities typically vary with position. Feature sensitivity changes are minimized for small motion tasks, and for configurations with large lens magnifications. However, if the feature sensitivity were constant, but coupled, the predicted path would still be straight-line motion irrespective of the number of degrees-offreedom. Since

$$
\underline{x}^{\circ}=J_{\text {feat }}^{-1} \underline{f}
$$

then

$$
\begin{aligned}
X_{i}^{\circ}= & \left(J_{\text {feat }-i, 1}^{-1} \Delta f_{1}+\ldots+J_{\text {feat }-i, n}^{-1} \Delta f_{n}\right)\left(1-e^{-t / \tau}\right) \\
= & K_{i}\left(1-e^{-t / \tau}\right)
\end{aligned}
$$

where $K_{i}$ is a constant. The constant relationship between any two Cartesian DOF becomes

$$
x_{i}^{0} / x_{j}^{0}=K_{i} / K_{j}
$$

which is the equation of a straight line.

Since we may not expect to find feature subsets which yield idealized sensitivity attributes, a feature selection strategy could seek a subset which best approximates these ideals; i.e., select features which minimize the coupling and sensitivity changes along a trajectory. in our research, the diagonal dominance measure, $D\left(J W_{p}\right)$, in eq. (9), is used to quantify system coupling. The feature selection strategy then becomes minimizing $D\left(J W_{p}\right)$ and $D\left(J_{f e a t}\right)$ over the set of candidate features. By minimizing $D\left(J W_{p}\right)$, improved dynamic response is achieved with SISO controllers. And, by minimizing $\mathrm{D}\left(\mathrm{J}_{\text {feat }}\right)$, closer to monotonic path performance may be expected. Each strategy may not produce mutually exclusive decisions, and arbitration between them would be based on the relative importance of each attribute. For example, a system could be feature uncoupled in the joint space of an articulated robot arm, but not uncoupled in Cartesian space. Since the degree-of-coupling plays such an important role in the independent control approach, our initial research has focused on evaluation of feature selection based on minimization of $D\left(J W_{p}\right)$.

\section{Simulation Studies}

Adaptive IBVS systems are highly nonlirear, making it difficult to analytically predict their potential periormance. Mainframe simulation studies were therefore used to evaluate IBVS control. The systems were modeled with progressively complex dynamics, kinematics, and feature coupling to understand their relative contributions to the control problem and system performance. Extensive studies have been completed for two and three DOF systems, and preliminary results are available for a 5 DOF system.

As an initial step in understanding image-based control, we studied visual servoing applications where the object and camera are stationary versus moving in the steady-state. Thus, step-input reference signals, defined by the "teach-byshowing" strategy, provide a suitable measure of system performance. The sampling period was varied from .003 to .1 seconds. The reference models are specified to be critically damped and with a bandwidth selected so that the sampling-tobandwidth ratio $\mathrm{f}_{\mathrm{s}} / \mathrm{f}_{\mathrm{BW}}$ was at least 20 . Faster sampling times were used to accentuate the magnitude of any nonlinear Coriolis and centrifugal dynamic terms. In practice, if the visual system processing requires relatively long sampling periods, then higher sampling rate minor-loop velocity feedback controllers would be required to compensate for Coulomb friction, structural resonances, and to eliminate any apparent complex plane poles.

To simplify the feature selection/assignment process, the lowfrequency, or $D C$ gain of $W_{p}$ is used to calculate the coupling index $D$ in equation (9). We observed that for a set of features, the feature/joint assignment remains constant over large regions of control for uncoupled kinematic configurations such as a Cartesian robot. For these configurations, off-line measurement of $\mathrm{JW}_{\mathrm{p}}$ in the initial image (i.e. by sequentially moving each degree-of-freedom by small increments, and measuring the change in features) can be used to select a fixed assignment over the entire control trajectory. Feature selection can also be based on initial image coupling if the potential candidates have relatively small coupling values. For coupled kinematic configurations, such an articulated arm, a feature/joint reassignment is predicted when controlling over large distances in space, and a fixed feature/joint assignment is only suitable for applications requiring small corrective motions. Some representative examples of adaptive IBVS control are discussed below.

One of the simulated systems included a dynamically nonlinear two DOF revolute joint articulated arm with a camera mounted to the second link. The task is to move the camera relative to a fixed line (or edge) in space. In these studies, the camera is modeled as a pinhole lens, and image features are derived from the idealized non-distorted two-dimensional image points. Image distortions are difficult to model and vary widely with lighting, transducer resolution and linearity. These conditions are manifested as measurement noise and modeled using varying levels of uniformly distributed noise added to the idealized extracted feature values. For this task, there are two independent control features: the perceived centroid and line length. The coupling index is used to resolve the feature/joint assignment. Ranges of task motions included examples with initial to desired position trajectories of short excursions (e.g. one inch), to long range motions over two feet, and over a broad range of arm configurations. The final or desired position of the camera was specified to be in close proximity to the line to

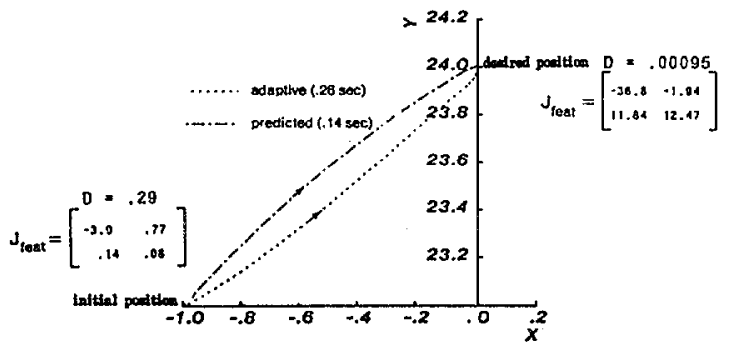

Figure 3-1: 2 DOF TASK 
accentuate rapidly changing feature sensitivities as the camera approaches the object. An example is shown in Figure 3-1 which displays the position trajectory of the camera and the corresponding rise-time. In Figure 3-1, the predicted trajectory is the path which would be achieved for perfect reference model following. The actual trajectory deviates from the predicted path due to initial transient identification errors which cannot be overcome in SISO control of the feature coupled system. The predicted trajectory deviates from a straight line by only .05 inches. For larger motion tasks, we observed that deviations of the predicted paths from a straight line remain on the same order of magnitude, even though the feature sensitivities are tightly coupled and vary dramatically over the trajectory. Actual path deviations from the predicted trajectories were acceptable for the larger motion tasks using a robot with linear uncoupled kinematics. However, with an articulated arm, the additional kinematic coupling leads to unacceptably large path deviations. These systems would require a coupled controller.

A three DOF configuration simulation consists of a stationary camera observing an object which is being moved. The object sits on top of a set of $X-Y-\theta$ translational and rotary stages. The task is to move the object relative to the fixed camera position. The extracted features available for polyhedral objects (e.g. cubes, pyramids, wedges, etc.) include the centroid and area of each visible plane, and the relative areas between any two adjacent planes. For this configuration there are more features available than there are degrees-of-freedom to control, and the coupling index is used to resolve both the feature selection and assignment. One method to evaluate the suitability of minimizing the coupling index to select features is to choose a very small motion task such that $J$, and thus $D\left(J W_{p}\right)$ stay essentially constant over the trajectory. System performance is evaluated for each possible combination of three features. For constant sensitivity the $X-Y$ stage predicted path is a straight line, and path performance is measured as the deviation from a straight line. Dynamic performance is measured as the position rise-time. Exhaustive testing shows that both path and dynamic performance improve as the coupling index decreases. For example, Figure 3-2 shows the path and rotation responses for three candidate features subsets when a cube is used as the object. For large motion tasks (e.g. trajectory greater than one foot), the observed paths approach straight lines as features are selected which reduce system coupling.

We have also have conducted preliminary evaluation of IBVS control using a 5 DOF system consisting of a camera mounted to the end of an articulated arm. We used fixed proportional controllers using trial and error gain adjustments and feature
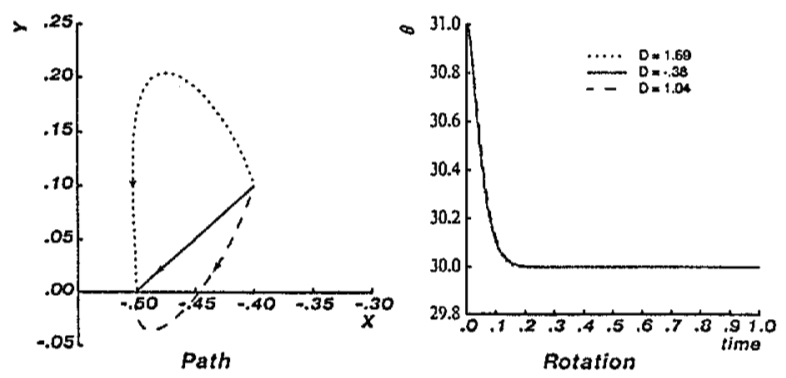

Figure 3-2: 3 DOF Examples assignment/selection to achieve stability with acceptable transient response and zero steady-state positional errors. While the predicted Cartesian paths were not derived, smooth joint motions resulted for properly tuned systems. It became difficult to tune the fixed controller for large motion trajectories because of the rapidly changing sensitivities and added kinematic coupling.

\section{Conclusion}

Our research has provided insight and analytical tools for the analysis, design, and evaluation of vision-based dynamic robot control systems. The evaluation of the adaptive image-based approach suggests that implementations of such systems may provide speed, accuracy, and simplified task training relative to position-based approaches. While IBVS control does not provide for explicit specification of the position trajectories, we have shown that smooth trajectories which approximate straight line motion can be achieved. For regimes of movement where image features have well-defined relations to the task, IBVS provides a useful complement to position-based control and might be used in a hierarchical framework to obtain an efficient implementation which incorporates both local and global models. In mobile robots this complementary IBVS/positionbased approach might be useful as a basis for control with respect to navigation features in an unstructured environment.

\section{References}

1. Sanderson, A. C., Weiss, L., "Adaptive Visual Servo Control of Robots", in Robot Vision, Pugh, A., ed., I.F.S. Publications Ltd., International Trends In Manufacturing Technology, 1983.

2. Weiss, Lee E., Dynamic Visual Servo Control of Robots: An Adaptive Image-Based Approach, PhD dissertation, Carnegie-Mellon University, 1984.

3. Coulon, P.Y., and Nougaret, M., "Use of a TV Camera System in Closed-Loop Position Control Mechanisms", in Robot Vision, Pugh, A., eds., I.F.S. Publications Ltd., International Trends in Manufacturing Technology, 1983.

4. Agin, G.J., "Real Time Control of a Robot with a Mobile Camera", Tech. report 179, SRI International, Feb. 1979.

5. Arthur C. Sanderson and Lee E. Weiss, "Image-Based Visual Servo Control of Robots', 26th Annual SPIE Technical Symposium, SPIE, August 1982.

6. Wylie, C.R., Advanced Engineering Mathematics, McGraw-Hill, 1961.

7. Neuman, C.P., Baradello, C.S., "Digital Transfer Functions for Microcomputer Control", IEEE Trans. on Systems, Man, and Cybernetics, Vol. Vol. SMC-9, No. 12, December 1979, pp. 856-860.

8. Chung, M.J., and Lee, C.S.G., "An adaptive Control Strategy for Computer-Based Manipulators", Tech. report 10-82, The Univ. of Michigan, Ann Arbor, August 1982.

9. Stone, H. and Neuman, C.P., "Dynamic Modeling and Adaptive Control of a Three DOF Robotic Manipultor", IEEE Transactions on Systems, Man, and Cybernetics, Vol. 14, July 1984. 
10. Dubowsky, S., and DesFOrges, D.T., "The Application of Model-Reference Adaptive Control to Robotic Manipulators', Trans. ASME, J.Dynamic Systems, Measurement, and Control, Vol. 101, Sept. 1979, pp. 193-200.

11. Horowitz, R., and Tomizuka, M., "An Adaptive Control Scheme for Mechanical Manipulators - Compensation of Nonlinearity and Decoupling Control", Winter Annual Meeting. Dynamics, Systems, and Control Div., ASME, Nov. 1980.

12. Koivo, A. J., Paul, R. P., "Manipulator with Self-Tuning Control", IEEE Conference on Cybernetics and Society, IEEE Systems, Man, and Cybernetics Society, 1980, pp. 1085-1089.

13. Koivo, A.J., and Guo, T.H., "Adaptive Linear Controller for Fobotic Manipulators", IEEE Trans, Automatic Control, Vol. AC-28, No. 2, Feb. 1983, pp. 162-171.

14. Le Borgne, M., Ibarra, J.M., and Espiau, B., "Adaptive Contro of High Velocity Manipulators", Proceedings of the 11th Intern. Symposium on Industrial Robots, Oct. 1981 , pp. 227.236.

15. Neuman, C.P. and Morris, R.L., "Classical Control Interpretation and Design of Microcomputer Adaptive Controllers", in Applications of Adaptive Control, Academic Press, 1980.

16. Neuman, C.P., and Stone, H.W., "MRAC Control of Robotic Manipulators", Proceedings of the Third Yale Workshop on Applications of Adaptive System Theory, June 1983.

17. Rosenbrock, H.H., Computer-Aided Control System Design, Academic Press, 1974. 